\title{
The Clinical Pharmacist's Role in Administering Pharmaceuticals for Hospitalized Patients
}

\author{
Asim Ahmed Elnour*
}

Department of Pharmacology, College of Medicine, UAE University, United Arab Emirates

Received: January 16, 2014, Accepted: January 18, 2014, Published: January 24, 2014

*Corresponding author: Asim Ahmed Elnour, Adjunct Clinical Associate Professor, Department of Pharmacology, College of Medicine, UAE University, United Arab Emirates, Tel: + 971-506-734096; E-mail: assahura1962@gmail.com

\section{Editorial}

In recent years, healthcare business is confronted with enormous challenges of quality-care health outcomes which may impose a significant impact on healthcare professionals, patients and healthcare management. The rapidly evolving era of new pharmaceuticals dictate an up-to-date expertise in handling, dispensing and administering the most safe, effective and economical pharmaceuticals.

The sprouting role of future pharmacist in monitoring these innovative pharmaceuticals such as biologic-amines, antiplatelets, anti-retroviral remedies, the super pills demand a new scope of clinical pharmacy services regarding administration of pharmaceuticals.

Nursing has ever taken the privilege of administering pharmaceuticals to patients in nursing homes and in hospitalized healthcare settings. Will it be a momentum of changing role to the nursing practice and an inevitable role to clinical pharmacists to take over, remains to be of paramount importance to the researchers to explore.

We may ask is it optimum and valued to learn the pharmacological basis and safe aspects of administering pharmaceuticals, in order to enable nursing competency in this ongoing process. The nursing is overwhelmed with twelve hours of performing diverse clinical tests, images and procedures. Nursing is quite expert in performing clinical monitoring of patients signs and symptoms, but not monitoring pharmaceuticals per se.

What about monitoring pharmaceuticals post-administration for adverse drug reactions, effectiveness and suitability for an individual patient. A clinical pharmacist is the most expert professional, who has comprehensive knowledge concerning all these integrated tasks.

We might argue that a nursing professional is taught to perform monitoring of the safety and effectiveness of the pharmaceuticals. Here again arises the issue of acquired learning (nursing) versus life-long knowledge (clinical pharmacist) as related to selection, monitoring, reviewing and reporting of the safety, effectiveness and pharmacoeconomic impact of the pharmaceuticals.

There is a dearth of research to delve further deeper into these challenging new paradigms and shift in emphasis for the clinical pharmacy profession. The pharmaceutical education and curriculum in phamacy colleges and pharmacists associations worldwide are countenance with these tasks.It remains to be another remarkable turning-point for the pharmacists, in conjunction with their new role in prescribing pharmaceuticals. 